CUBO A Mathematical Journal

Vol.12, № 03, (203-212). October 2010

\title{
Analytic Continuation and Applications of Eigenvalues of Daubechies' Localization Operator
}

\author{
KUnio Yoshino \\ Department of Natural Sciences, Faculty of Knowledge Engineering, \\ Tokyo City University, Tokyo 158-8557, Japan \\ email: yoshinok@tcu.ac.jp
}

\begin{abstract}
In this paper we introduce generating functions of eigenvalues of Daubechies' localization operator, study their analytic properties and give analytic continuation of these eigenvalues. Making use of generating functions, we establish a reconstruction formula of symbol functions of Daubechies' localization operator with rotational invariant symbols.
\end{abstract}

\section{RESUMEN}

Introducimos funciones generadas por los autovalores del operador de localización de Daubechies, estudiamos sus propiedades analíticas y damos continuación analítica de los autovalores. Haciendo uso de las funciones generadas, establecemos la fórmula de reconstrucción de funciones símbolo del operador de localización de Daubechies con símbolos rotacional invariante.

Key words and phrases: Hermite functions, Daubechies (localization) operator, Borel transform, asymptotic expansion.

Math. Subj. Class.: 33, 44, 46F. 


\section{Introduction}

The Daubechies (localization) operator was introduced by Ingrid Daubechies in [4], where she mainly treated localization operators with rotational invariant symbols. In particular, she expressed eigenvalues as Mellin transforms of symbol functions. She also proved that Hermite functions are eigenfunctions of localization operators with rotational invariant symbols. So far, the theory of localization operators has been studied by several researchers in various fields ([2], [5], [7], [10], [11], [12]).

In this paper we will study analytic properties of generating functions of eigenvalues of Daubechies' localization operators. We will also give an analytic continuation of eigenvalues of Daubechies' localization operator. Making use of generating functions, we will establish the reconstruction formula of symbol functions of Daubechies' localization operators with rotational invariant symbol.

For the simplicity, we will confine ourselves to the 1-dimensional case in this paper. In section 2 we will introduce Daubechies' localization operator. In section 3 we will give the analytic continuation of eigenvalues of Daubechies' localization operator. In section 4 we will define the generating function of eigenvalues. In final section 5 we will establish the reconstruction formulas for rotational invariant symbol function.

\section{Daubechies' Localization Operator}

According to [4], we define the localization operator $P_{F}$ as follows.

Definition 1 ([4]). Daubechies' localization operator is

$$
P_{F}(f)(x)=(2 \pi)^{-1} \iint_{\mathbb{R}^{2}} F(p, q) \phi_{p, q}(x)<\phi_{p, q}, f>d p d q,
$$

where $F(p, q) \in L^{1}\left(\mathbb{R}^{2}\right), f(x) \in L^{2}(\mathbb{R})$

$$
\phi_{p, q}(x)=\pi^{-1 / 4} e^{i p x} e^{-(x-q)^{2} / 2},
$$

and $<\phi_{p, q}, f>$ denotes the inner product

$$
\int_{\mathbb{R}} \overline{\phi_{p, q}(x)} f(x) d x .
$$

The function $F(p, q)$ is called the symbol function of the operator $P_{F}$.

Daubechies obtained the following results. 
Proposition 1 ([4]). Suppose that $F(p, q) \in L^{1}\left(\mathbb{R}^{2}\right)$. Then

(i) If $F(p, q) \geq 0$, then $P_{F}$ is a positive operator.

(ii) $P_{F}$ is bounded operator. that is,

$$
\left\|P_{F}(f)\right\|_{L^{2}} \leq(2 \pi)^{-1 / 2}\|f\|_{L^{2}}\|F\|_{L^{1}}, \quad\left(f \in L^{2}(\mathbb{R})\right) .
$$

(iii) $P_{F}$ is a trace class operator.

Proposition 2 ([4]). Suppose

$$
F(p, q)=\tilde{F}\left(r^{2}\right), \quad \text { where } r^{2}=p^{2}+q^{2} .
$$

Then

(i) The Hermite functions $h_{m}(x)$ are eigenfunctions of the operator $P_{F}$ :

$$
P_{F}\left(h_{m}\right)(x)=\lambda_{m} h_{m}(x), \quad m \in \mathbb{N} .
$$

(ii) Secondly,

$$
\lambda_{m}=\frac{1}{m !} \int_{0}^{\infty} e^{-s} s^{m} \tilde{F}(2 s) d s, \quad m \in \mathbb{N},
$$

where the Hermite functions $h_{m}(x)$ are defined by

$$
h_{m}(x)=(-1)^{m}\left(2^{m} m ! \sqrt{\pi}\right)^{-1 / 2} \exp \left(x^{2} / 2\right) \frac{d^{m}}{d x^{m}} \exp \left(-x^{2}\right), \quad m \in \mathbb{N} .
$$

For details on Hermite functions, we refer the reader to $[6,7,10]$.

In what follows we assume that

(i) $F(p, q) \in L^{1}\left(\mathbb{R}^{2}\right)$.

(ii) $F(p, q)$ is rotational invariant, that is,

$$
F(p, q)=\tilde{F}\left(r^{2}\right), \quad \text { where } \quad r^{2}=p^{2}+q^{2} .
$$

\section{Analytic Continuation of Eigenvalues of Daubechies Operator}

In this section we consider the analytic continuation of the eigenvalues $\left\{\lambda_{m}\right\}_{m=0}^{\infty}$. By Proposition 2 we have

$$
\lambda_{m}=\frac{1}{m !} \int_{0}^{\infty} e^{-s} s^{m} \tilde{F}(2 s) d s .
$$


We put

$$
\lambda(z)=\frac{1}{\Gamma(z+1)} \int_{0}^{\infty} e^{-s} s^{z} \tilde{F}(2 s) d s, \quad z \in \mathbb{C}, \operatorname{Re}(z)>0 .
$$

where $\Gamma(z)$ is Euler's Gamma function.

Then we have the following proposition.

Proposition 3. $\lambda(z)$ have following properties:

(i) $\lambda(z)$ is holomorphic in the right half plane $\operatorname{Re}(z)>0$.

(ii) There exists a positive constant $C$ such that

$$
|\lambda(z)| \leq \frac{C}{\sqrt{|z|}} e^{\frac{\pi}{2}|y|}, \quad z=x+i y \in \mathbb{C}, x>0 .
$$

(iii) $\lambda(z)$ interpolates the eigenvalues $\left\{\lambda_{m}\right\}_{m=0}^{\infty}$, that is,

$$
\lambda(m)=\lambda_{m}, \quad m \in \mathbb{N}
$$

(iv) There exists a positive constant $C$ such that

$$
\left|\lambda_{m}\right| \leq \frac{C}{\sqrt{|m|}}, \quad m \in \mathbb{N} .
$$

Proof. The proof is as follows.

(i) We can prove the holomorphicity of $\lambda(z)$ by Morea's theorem and Lebesgue's dominated convergence theorem.

(iii) is obvious.

(iv) follows from (ii) and (iii).

So we will prove statement (ii). By Stirling's formula,

$$
\Gamma(z+1) \sim z^{z} e^{-z} \sqrt{2 \pi z}, \quad \operatorname{Re}(z)>0
$$


and $e^{-s} s^{x} \leq e^{-x} x^{x}$ for $s \geq 0$, we have

$$
\begin{aligned}
|\lambda(z)| & =\left|\frac{1}{\Gamma(z+1)} \int_{0}^{\infty} e^{-s} s^{z} \tilde{F}(2 s) d s\right| \\
& \leq \frac{1}{|\Gamma(z+1)|} \int_{0}^{\infty} e^{-s}\left|s^{z}\right||\tilde{F}(2 s)| d s \\
& \leq \frac{C}{\left|z^{z} e^{-z}\right| \sqrt{2 \pi|z|} \mid} \int_{0}^{\infty} e^{-s} s^{x}|\tilde{F}(2 s)| d s \\
& \leq \frac{C e^{y \arg (z)}}{x^{x} e^{-x} \sqrt{2 \pi|z|}} \int_{0}^{\infty} e^{-x} x^{x}|\tilde{F}(2 s)| d s \\
& \leq \frac{C}{\sqrt{2 \pi|z|}} e^{\frac{\pi}{2}|y|} \int_{0}^{\infty}|\tilde{F}(2 s)| d s \\
& \leq \frac{C^{\prime}}{\sqrt{|z|}} e^{\frac{\pi}{2}|y|} .
\end{aligned}
$$

Remark 1. The function $\lambda(z)$ is the unique analytic continuation of eigenvalues $\left\{\lambda_{m}\right\}_{m=0}^{\infty}$ because of (ii) in Proproposition 3 and Carlson's theorem [3].

\section{Generating Functions of Eigenvalues of Daubechies Op- erator}

In this section we introduce two generating functions $\Lambda(w)$ and $G(t)$ of the eigenvalues $\left\{\lambda_{m}\right\}_{m=0}^{\infty}$. We begin with $\Lambda(w)$. Put

$$
\Lambda(w)=\sum_{m=0}^{\infty} \lambda_{m} w^{m}, \quad(|w|<1) .
$$

Due to (iv) in Proposition 3, the right-hand side is a convergent series if $|w|<1$.

We will show some analytic properties of $\Lambda(w)$.

Proposition 4. Suppose that $\left\{\lambda_{m}\right\}_{m=0}^{\infty}$ are eigenvalues of $P_{F}$. Then

(i) The function $\Lambda(w)$ is given by the integral

$$
\Lambda(w)=\int_{0}^{\infty} e^{-s(1-w)} \tilde{F}(2 s) d s, \quad \operatorname{Re}(w)<1 .
$$

(ii) $\Lambda(w)$ is holomorphic in the left-half plane $\{w \in \mathbb{C}: \operatorname{Re}(w)<1\}$ and is bounded in its closure $\{w \in \mathbb{C}: \operatorname{Re}(w) \leq 1\}$.

(iii) $\Lambda(i v) \in C_{0}(\mathbb{R})$ for $v \in \mathbb{R}$, that is, $\Lambda(i v) \in C(\mathbb{R})$ and $\lim _{|v| \rightarrow \infty} \Lambda(i v)=0$. 
Proof. We prove the three parts.

(i) By (ii) in Proposition 2, we have

$$
\begin{aligned}
\Lambda(w) & =\sum_{m=0}^{\infty} \lambda_{m} w^{m} \\
& =\sum_{m=0}^{\infty} \frac{w^{m}}{m !} \int_{0}^{\infty} e^{-s} s^{m} \tilde{F}(2 s) d s \\
& =\int_{0}^{\infty} e^{-s} \tilde{F}(2 s) \sum_{m=0}^{\infty} \frac{(w s)^{m}}{m !} d s \\
& =\int_{0}^{\infty} e^{-s(1-w)} \tilde{F}(2 s) d s .
\end{aligned}
$$

(ii) For $\operatorname{Re}(w) \leq 1$, we have

$$
|\Lambda(w)| \leq \int_{0}^{\infty}\left|e^{-s(1-w)}\left\|\tilde{F}(2 s)\left|d s \leq \int_{0}^{\infty}\right| \tilde{F}(2 s) \mid d s=\right\| \tilde{F} \|_{L^{1}} .\right.
$$

(iii) $\Lambda(i v)$ is the Fourier transform of the $L^{1}$ function $e^{-s} \tilde{F}(2 s)$, for $s \geq 0$. Hence it belongs to $C_{0}\left(\mathbb{R}^{n}\right)$ by the Riemann-Lebesgue theorem [9].

Proposition 5. Suppose that $F(p, q)$ is positive. If

$$
\limsup _{m \rightarrow \infty} \lambda_{m}^{1 / m}=1,
$$

then $w=1$ is a singular point of $\Lambda(w)$.

Proof. Since $F(p, q)$ is positive, then $P_{F}$ is a positive operator by Proposition 1 . Therefore, all the eigenvalues of $P_{F}$ are nonnegative. By the Cauchy-Hadamard formula, the radius of convergence of the power series

$$
\sum_{m=0}^{\infty} \lambda_{m} w^{m}
$$

is 1 . By Vivanti's theorem, $w=1$ is a singular point of $\Lambda(w)$.

Proposition 6. Suppose the support of $\tilde{F}(2 s)$ is contained in $[0, a]$. Then there exists a positive constant $C$ such that

(i) $\left|\lambda_{m}\right| \leq C \frac{a^{m}}{m !}, \quad m \in \mathbb{N}$.

(ii) $\Lambda(w)$ is an entire function of exponential type.

Proof. We prove the two parts of the proposition. 
(i) Since the support of $\tilde{F}(2 s)$ is contained in the closed interval $[0, a]$, by (ii) in Proposition 2 , we have

$$
\begin{aligned}
\lambda_{m} & =\frac{1}{m !} \int_{0}^{a} e^{-s} \tilde{F}(2 s) s^{m} d s \\
& \leq \frac{a^{m}}{m !} \int_{0}^{a}|\tilde{F}(2 s)| d s .
\end{aligned}
$$

Therefore, the inequality $\left|\lambda_{m}\right| \leq C \frac{a^{m}}{m !}$ is valid.

(ii) Since

$$
\begin{aligned}
|\Lambda(w)| & \leq \int_{0}^{a}|\tilde{F}(2 s)| e^{-s(1-u)} d s \\
& \leq e^{a|u|} \int_{0}^{a}|\tilde{F}(2 s)| d s,
\end{aligned}
$$

we have

$$
|\Lambda(w)| \leq C e^{a|u|}, \quad w=u+i v \in \mathbb{C} .
$$

Now we consider following formal power series:

$$
\sum_{m=0}^{\infty} m ! \lambda_{m} t^{-m-1}
$$

In general, the series on right-hand side is divergent. But this formal power series is an asymptotic expansion of the Hilbert transform of $\tilde{F}(2 s) e^{-s}$. Namely, if we put

$$
G(t)=\int_{0}^{\infty} \frac{\tilde{F}(2 s) e^{-s}}{t-s} d s, \quad t \in \mathbb{C} \backslash[0, \infty]
$$

then $G(t)$ has following properties.

Proposition 7. For the function $G(t)$ we have

(i) $G(t)$ is Laplace transform of $\Lambda(w)$.

(ii) $\sum_{m=0}^{\infty} m ! \lambda_{m} t^{-m-1}$ is an asymptotic expansion of $G(t)$.

\section{Proof.}


(i) By (ii) in Proposition $4, \Lambda(w)$ is bounded in left-half plane. So we can consider the Laplace transform of $\Lambda(w)$ along the negative real axis:

$$
\begin{aligned}
\int_{0}^{-\infty} \Lambda(w) e^{-t w} d w & =\int_{0}^{-\infty}\left\{\int_{0}^{\infty} e^{-s(1-w)} \tilde{F}(2 s) d s\right\} e^{-t w} d w \\
& =\int_{0}^{\infty} \tilde{F}(2 s) e^{-s}\left\{\int_{0}^{-\infty} e^{w(s-t)} d w\right\} d s \\
& =\int_{0}^{\infty} \frac{\tilde{F}(2 s) e^{-s}}{t-s} d s \\
& =G(t), \quad \text { for } \operatorname{Re} t<0 .
\end{aligned}
$$

(ii) Secondly,

$$
\begin{aligned}
G(t) & =\int_{0}^{\infty} \frac{\tilde{F}(2 s) e^{-s}}{t-s} d s \\
& =\frac{1}{t} \int_{0}^{\infty} \frac{\tilde{F}(2 s) e^{-s}}{1-s t^{-1}} d s \\
& =\frac{1}{t} \int_{0}^{\infty} \tilde{F}(2 s) e^{-s}\left\{\sum_{m=0}^{N}\left(s t^{-1}\right)^{m}+\frac{\left(s t^{-1}\right)^{N+1}}{1-s t^{-1}}\right\} d s \\
& =\sum_{m=0}^{N} m ! \lambda_{m} t^{-m-1}+\frac{1}{t^{N+1}} \int_{0}^{\infty} \frac{\tilde{F}(2 s) e^{-s} s^{N+1}}{t-s} d s .
\end{aligned}
$$

Hence if $|t| \geq R$ and $0<\delta \leq \arg (t) \leq 2 \pi-\delta$, then we have

$$
\begin{aligned}
\left|G(t)-\sum_{m=0}^{N} m ! \lambda_{m} t^{-m-1}\right| & \leq \frac{(N+1) !}{R \sin \delta} \frac{\lambda_{N+1}}{|t|^{N+1}} \\
& \leq C \frac{N ! \sqrt{N+1}}{R \sin \delta|t|^{N+1}} .
\end{aligned}
$$

Proposition 8. Suppose that support of $\tilde{F}(2 s)$ is contained in $[0, a]$. Then

(i) $G(t)$ is holomorphic in $\mathbb{C} \backslash[0, a]$.

(ii) $\sum_{m=0}^{\infty} m ! \lambda_{m} t^{-m-1}$ converges in $|t|>a$.

\section{Proof.}

(i) From the assumption on the support of $\tilde{F}(2 s)$, we have

$$
G(t)=\int_{0}^{a} \frac{\tilde{F}(2 s) e^{-s}}{t-s} d s .
$$

So $G(t)$ is holomorphic in $\mathbb{C} \backslash[0, a]$. 
(i) By (i) in Proposition 6,

$$
\left|\lambda_{m}\right| \leq C \frac{a^{m}}{m !}, \quad m \in \mathbb{N} .
$$

Hence

$$
\sum_{m=0}^{\infty} m ! \lambda_{m} t^{-m-1}
$$

converges if $|t|>a$.

Remark 2. The function $\Lambda(w)$ is the Borel transform of $G(t)$. For details on the Borel and Hilbert transforms, we refer the reader to [1, 8, 9].

\section{Reconstruction of Symbol Functions}

In this section we establish our main results.

Theorem 1. The function

$$
\tilde{F}(2 s)=(2 \pi)^{-1} e^{s} \mathfrak{F}(\Lambda(i v))(s),
$$

is valid in distribution sense, where

$$
\mathfrak{F}(\Lambda(i v))=\int_{-\infty}^{\infty} e^{-i s v)} \Lambda(i v) d v
$$

is Fourier transform of $\Lambda(i v)$.

Proof. By (i) in Proposition 4, we have

$$
\begin{aligned}
\Lambda(i v) & =\int_{0}^{\infty} e^{-s(1-i v)} \tilde{F}(2 s) d s \\
& =\int_{0}^{\infty} e^{i s v} e^{-s} \tilde{F}(2 s) d s
\end{aligned}
$$

This means that $\Lambda(i v)$ is the inverse Fourier transform of $e^{-s} \tilde{F}(2 s)$. Since $\tilde{F}(2 s)$ is an $L^{1}$ function, then $e^{-s} \tilde{F}(2 s)$ is a tempered distribution. Hence, as tempered distribution, we have

$$
\tilde{F}(2 s)=e^{s} \mathfrak{F}(\Lambda(i v))(s)
$$

Theorem 2. The function $\tilde{F}(2 s)$ is given by the formula

$$
\tilde{F}(2 s)=e^{s} \lim _{t \rightarrow 0} \frac{-1}{2 \pi i}(G(s+i t)-G(s-i t)) .
$$


Proof. It is well known that the boundary value

$$
\lim _{t \rightarrow 0} \frac{-1}{2 \pi i}[G(s+i t)-G(s-i t)]
$$

is the inverse map of the Hilbert transform [8]. Since $G(t)$ is Hilbert transform of $e^{-s} \tilde{F}(2 s)$, we have

$$
\lim _{t \rightarrow 0} \frac{-1}{2 \pi i}(G(s+i t)-G(s-i t))=e^{-s} \tilde{F}(2 s)
$$

\section{References}

[1] Anderson, M., Topics in Complex Analysis, Springer-Verlag, New York, Berlin, Heidelberg, 1996.

[2] Ashino, R., Boggiatto, P. And Wong, M.W., Advanced in Pseudo-Differential Operators, Birkhäuser-Verlag, Basel, Berlin, Boston, 2000.

[3] BoAs, R.P., Entire Functions, Academic Press, New York, 1954.

[4] Daubechies, I., A time frequency localization operator; A geometric phase space approach, IEEE. Trans. Inform. Theory, vol.34, pp. 605-612, 1988.

[5] Daubechies, I., Ten Lectures on Wavelets, Rutgers University and AT \& T Bell Laboratories, 1992.

[6] Folland, G.B., Harmonic Analysis in Phase Space, Princeton Univ. Press, 1989.

[7] Gröhenig, K., Foundations of Time-Frequency Analysis, Birkhäuser-Verlag, Basel, Berlin, Boston, 2000.

[8] Morimoto, M., An Introduction to Sato's Hyperfunction, Translation of Math. Monograph, vol. 129, Amer. Math. Soc. Providence, Rhode Island, 1993.

[9] Rudin, W., Real and Complex Analysis, McGraw-Hill Book Company, New York, 1987.

[10] Wong, M.W., Weyl Transforms, Springer-Verlag, New York, 1998.

[11] Wong, M.W., Localization Operators on the Weyl-Heisenberg Group, Geometry, Analysis and Applications, Proceedings of the International Conference (editor:P.S.Pathak), pp. 303-314, 2001.

[12] Wong, M.W., Wavelet Transforms and Localization Operator, Birkhäuser-Verlag, Basel, Berlin, Boston, 2002. 